\title{
FACTORES DE RIESGO DE EXPOSICIÓN DURANTE EL MANEJO Y USO DE PLAGUICIDAS EN FUMIGADORES URBANOS
}

\author{
José Francisco HERRERA MORENO ${ }^{1,2}$, Alma Betsaida BENITEZ TRINIDAD ${ }^{1,2}$, \\ Maria del Carmen XOTLANIHUA GERVACIO ${ }^{1,2}$, Yael Yvette BERNAL HERNÁNDEZ ${ }^{1}$, \\ Irma Martha MEDINA DÍAZ ${ }^{1}$, Briscia Socorro BARRÓN VIVANCO ${ }^{1}$, \\ Cyndia Azucena GONZÁLEZ ARIAS ${ }^{1}$, Norma Elena PÉREZ HERRERA ${ }^{3}$ y \\ Aurora Elizabeth ROJAS GARCÍA ${ }^{1 *}$
}

${ }^{1}$ Laboratorio de Contaminación y Toxicología Ambiental, Secretaría de Investigación y Posgrado, Universidad Autónoma de Nayarit, Ciudad de la Cultura s/n, Col. Centro, 63000 Tepic, Nayarit, México

${ }^{2}$ Posgrado en Ciencias Biológico Agropecuarias, Unidad Académica de Agricultura, km. 9 Carretera TepicCompostela, Xalisco, Nayarit, México

${ }^{3}$ Unidad Interinstitucional de Investigación Clínica y Epidemiológica, Facultad de Medicina, Universidad Autónoma de Yucatán, Mérida, México

*Autor para correspondencia: erojas@uan.edu.mx

(Recibido enero 2017; aceptado febrero 2018)

Palabras clave: plaguicidas, prácticas de uso y manejo de plaguicidas, fumigadores

\section{RESUMEN}

Los plaguicidas son compuestos ampliamente utilizados para el control de plagas en diversos sectores, y una de las poblaciones con mayor riesgo de exposición ocupacional a estos xenobióticos son los fumigadores urbanos. El objetivo del presente trabajo fue conocer los factores de riesgo de exposición a plaguicidas relacionados con las prácticas de su uso y manejo en una población de fumigadores de Nayarit, México. Se realizó un estudio transversal, descriptivo y analítico en trabajadores que se dedican a la fumigación de casas, escuelas y otras áreas. De acuerdo con la información obtenida a través de un cuestionario estructurado, el $31.3 \%$ de los participantes realiza actividades de abatización, el $63.2 \%$ mezcla y aplica plaguicidas, y el $5.5 \%$ restante realiza otras actividades relacionadas con plaguicidas. Más del $85 \%$ no usa el equipo de protección personal adecuado. Los organofosforados (OF) son los plaguicidas más usados por los trabajadores, seguidos de los piretroides y carbamatos. El $11.1 \%$ de los fumigadores se ha intoxicado por lo menos en una ocasión y el $31 \%$ refiere no saber qué es una intoxicación, mientras que el $33.3 \%$ de la población no considera su ocupación peligrosa y el $46.9 \%$ refiere no haber recibido capacitación previa para el manejo de plaguicidas. Los datos obtenidos en este trabajo sugieren un riesgo importante de exposición a plaguicidas en la población de estudio debido al desconocimiento de las medidas de seguridad adecuadas o falta de uso de las mismas, o bien a que un importante porcentaje de la población no cuenta con el equipo necesario; asimismo, como resultado de las condiciones climáticas en la zona de trabajo, las cuales dificultan su empleo, por la incomodidad que representa el uso del equipo, de la falta permanente de capacitación y, sobre todo, de la ausencia de concientización sobre el riesgo que conlleva un mal uso y manejo de plaguicidas.

Key words: pesticides, use practices and handling of pesticides, sprayers 


\begin{abstract}
Pesticides are compounds widely used for pest control in different sectors; urban sprayers represent one of the populations with higher risk of occupational exposure to these xenobiotics. The aim of this work was to evaluate the practices of use and handling of pesticides, which involve a risk of exposure in a population of urban sprayers from Nayarit, Mexico. A cross-sectional, descriptive, analytical study was conducted in workers engaged in fumigation of houses, schools and other areas. According to the gathered information from participants, $31.3 \%$ carries out activities of abatization, $63.2 \%$ mixes and applies pesticides, and $5.5 \%$ performes other activities related with pesticide. Over $85 \%$ does not use proper personal protective equipment. Organophosphorus (OP), followed by pyrethroids and carbamates, were mainly used by participants. About $11.1 \%$ of sprayers had been intoxicated and $31 \%$ did not have knowledge on whether they have manifested poisoning. Further, $33.3 \%$ of the population does not consider pesticide spraying activity as a hazardous occupation, and $46.9 \%$ did not received any training in pesticide management. The data obtained in this study suggest a significant risk of exposure to pesticides in the study population, due to the lack of knowledge regarding adequate safety measures or lack of the necessary personal protective equipment, adverse weather conditions while working, the discomfort that represents the use of the equipment, and/or a permanent lack of training on the proper use and handling of pesticides.
\end{abstract}

\section{INTRODUCCIÓN}

El uso de plaguicidas en México se ha fomentado ampliamente para el control y combate de vectores que transmiten enfermedades víricas como chikungunya, dengue, paludismo y zika, las cuales actualmente constituyen un problema de salud pública en México (Sánchez-González et al. 2016).

De acuerdo con la Organización de las Naciones Unidas para la Agricultura y la Alimentación (FAO), un plaguicida es cualquier sustancia o mezcla de sustancias químicas o biológicas destinada a prevenir, destruir o controlar cualquier plaga, incluyendo vectores de enfermedades, especies no deseadas de plantas o animales que causen perjuicios o que interfieren de cualquier otra forma en la producción agropecuaria o forestal (FAO 2014). Estos agentes químicos incluyen una gran variedad de compuestos diferentes en su composición, propiedades fisicoquímicas y toxicidad (March 2014). Por su origen, estos productos se clasifican en naturales-botánicos (extractos de plantas), biológicos (virus o microorganismos), inorgánicos (minerales) y sintéticos (COFEPRIS 2015). Si bien existen estudios que mencionan los efectos adversos producidos por estos compuestos en el ser humano, son pocos los que describen los efectos en los empleados dedicados a la fumigación industrial, doméstica, de servicios turísticos y de salud. Estos trabajadores pueden ser especialmente vulnerables si se considera que están expuestos desde la preparación hasta su aplicación.

Por otro lado, de acuerdo con la Organización Panamericana de la Salud y la Organización Mundial de la Salud (OPS/OMS 2002), se ha estimado que se producen tres millones de intoxicaciones agudas por plaguicidas cada año y de 80000 a 220000 defunciones; una tasa de letalidad de $0.25 \%$ para las intoxicaciones en los países desarrollados y $0.5 \%$ en los países en desarrollo. En América Central la tasa de incidencia de intoxicaciones por plaguicidas es de 35 por cada 100000 habitantes y 17.8 por cada 100000 son de origen ocupacional.

En este sentido se ha estimado que el uso ocupacional de plaguicidas produce 355000 muertes. Dos factores que contribuyen son la falta de conocimiento sobre las medidas de seguridad y la reutilización de los envases vacíos (López et al. 2008).

De acuerdo con la FAO, en México, se han usado en promedio 4.55 toneladas de plaguicidas en una densidad territorial de 1000 hectáreas entre 2009 y 2010. Concretamente en el caso de insecticidas, en 2014 se emplearon 32406 toneladas (FAO 2016). Debido a la falta de monitoreo en México, no se cuenta con información detallada sobre el patrón de uso de estos compuestos, ni de un panorama completo de los efectos que generan (Arellano-Aguilar y Rendón 2016). En este sentido, Martínez-Valenzuela y Gómez-Arroyo (2007) han calculado que se producen entre 500000 y 1000000 
de intoxicaciones agudas anuales y entre $500 \mathrm{y}$ 20000 defunciones.

\section{Plaguicidas: percepción de riesgo, manejo y uso}

Las poblaciones expuestas ocupacionalmente a plaguicidas - como los fumigadores urbanos - son grupos vulnerables que requieren de capacitación en el uso y manejo de estos compuestos y, más aún, sobre la prevención de accidentes laborales. En México, de acuerdo con la legislación inscrita en la Constitución Política de los Estados Unidos Mexicanos, específicamente en la Ley Federal del Trabajo, corresponde a la Secretaría de Salud y a la Comisión Federal para la Protección Contra Riesgos Sanitarios (COFEPRIS), realizar acciones como campañas informativas sobre los riesgos del uso de plaguicidas, así como fomentar el uso de equipo de protección personal y prácticas de seguridad durante la exposición laboral (Sánchez-Guerra et al. 2011). Diferentes tareas, como la preparación de mezclas, aplicación y almacenamiento de plaguicidas, son las que pueden representar un riesgo potencial en poblaciones ocupacionalmente expuestas a estos compuestos. A lo anterior se suman las condiciones socioambientales adversas, el uso de equipos y vestimentas inapropiadas, el uso de equipos en mal estado y la falta de capacitación adecuada (López et al. 2008), así como una baja percepción del riesgo vinculado al manejo de este tipo de sustancias por parte de los trabajadores. La percepción de riesgo puede definirse como el juicio emitido de manera subjetiva que hacen las personas sobre las características y severidad de un peligro, principalmente con referencia a amenazas naturales y al ambiente o la salud (Berube 2009).

El objetivo del presente trabajo fue conocer los factores de riesgo de exposición a plaguicidas relacionados con las prácticas de uso y manejo en una población de fumigadores en Nayarit.

\section{MATERIALES Y MÉTODOS}

\section{Diseño de estudio}

Se realizó un estudio transversal, descriptivo y analítico en 208 trabajadores, 186 de ellos dedicados a la fumigación de casas, escuelas y otras áreas, en el estado de Nayarit, México. Los participantes fueron identificados a través de los registros actuales de la Secretaría de Salud del estado. La información sobre las características socioeconómicas, antropométricas, edad, dieta, hábitos nocivos (consumo de drogas, tabaco y alcohol), exposición a plaguicidas y percepción de riesgo, se evaluaron mediante un cuestionario estructurado. La percepción del riesgo se evaluó a través de reactivos como los siguientes: si consideraban su ocupación peligrosa para su salud, si creían que el usar productos químicos representaba algún peligro o daño, si conocían el significado de los colores de las etiquetas de los envases de plaguicidas (concretamente cuál color en las etiquetas representaba mayor y menor peligro), entre otros. Previo al uso del cuestionario, se realizó un estudio piloto para evaluar la validez de los reactivos y se hicieron las modificaciones para adecuarlos al contexto sociocultural de la población de estudio. También se evaluaron algunas variables clínicas por cuestionario (presencia de anemia, padecimientos crónicos como diabetes, entre otros), así como por pruebas de laboratorio. Los datos mostraron una correlación positiva y significativa entre la información obtenida por encuesta y por pruebas de laboratorio (Benítez-Trinidad et al. 2018).

Como criterios de inclusión al estudio se consideraron individuos mayores de 18 años y que aceptaran participar en él.

\section{Estratificación de grupos de estudio}

La población de estudio se estratificó en tres grupos de acuerdo con la intensidad de la exposición, la cual se evaluó de manera retrospectiva a través de un cuestionario. En éste se consideró la actividad laboral específica, el uso de protección personal, tiempo de exposición y actividades adicionales en campo, entre otras. Además, como biomarcador de exposición se determinó la actividad de la enzima butirilcolinesterasa $(\mathrm{BuChE})$. Los tres grupos considerados fueron:

- Grupo de referencia: individuos que trabajaban en oficina, no realizaban actividades de fumigación, no trabajaban de manera permanente ni temporal en el campo, con un rango de actividad de $\mathrm{BuChE}$ de 4937-8922 U/L.

- Grupo de exposición moderada: individuos que realizaban actividades de abatización, algunos fumigaban ocasionalmente y además podían tener exposición en el campo ocasionalmente. Los participantes tuvieron un rango de actividad de $\mathrm{BuChE}$ de 1377-9329 U/L. Este grupo tenía un amplio rango de exposición. El principal plaguicida que utilizaban era temefos y en menor proporción clorpirifos.

- Grupo de exposición alta: individuos que participaban en actividades de fumigación utilizando vehículos, moto mochila o bomba Hudson. Algunos también fumigaban en el campo. El rango de actividad de BuChE fue de 871-7418 U/L. Este 
grupo estaba expuesto principalmente a clorpirifos y deltametrina, entre otros.

\section{Determinación de actividad de BuChE}

$\mathrm{La}$ actividad de $\mathrm{BuChE}$ se determinó en muestras de suero utilizando el método de Worek et al. (1999) con algunas modificaciones. La mezcla de reacción consistió en $10 \mu \mathrm{L}$ de suero, $3 \mathrm{~mL}$ de buffer de fosfato (0.1 M, pH 7.4) y $100 \mu \mathrm{L}$ de DTNB $10 \mathrm{mM}$. La mezcla se incubó a $37^{\circ} \mathrm{C}$ durante 10 min y se añadieron $50 \mu \mathrm{L}$ de yoduro de butiriltiocolina $(63.2 \mathrm{mM})$. El cambio de absorbancia se monitoreó a $405 \mathrm{~nm}$ cada minuto durante 4 min en un espectrofotómetro Genesys 10Bio (Wisconsin, USA). La actividad BuChE se expresó en U/L.

\section{Manejo de los aspectos bioéticos}

A todos los participantes se les explicó el objetivo del estudio y se les invitó a participar de manera voluntaria en el mismo. A las personas que aceptaron se les solicitó que firmaran una carta de consentimiento informado. Este estudio de investigación forma parte de un macroproyecto que ha sido aprobado por la Comisión Estatal de Bioética de Nayarit (CEBN/0112017).

\section{Análisis estadístico}

Se generó una base constituida por las variables obtenidas de todos los instrumentos y datos analíticos. Una vez realizada la captura y limpieza de los datos, se procedió a realizar el análisis descriptivo, frecuencias y proporciones. Para el análisis de las variables categóricas se utilizó la prueba de ji cuadrado y para las variables continuas se utilizaron las pruebas de Mann-Whitney y Kruskal-Wallis. Los valores de $\mathrm{p}<0.05$ se consideraron estadísticamente significativos. Los análisis estadísticos se desarrollaron utilizando el programa Stata v. 14.0 (Stata Statistical Software, Stata Corporation, College Station, Texas).

\section{RESULTADOS}

\section{Características generales de la población}

La población de estudio consistió en 208 participantes, de los cuales 69 fueron mujeres y 139 hombres. La media geométrica de la edad poblacional fue de 36.9 años con un rango de 20 a 68 años. En cuanto al índice de masa corporal (IMC), la media geométrica de la población fue de $29.3 \mathrm{~kg} / \mathrm{m}^{2}$, con un rango de 19 a $54.4 \mathrm{~kg} / \mathrm{m}^{2}$. De acuerdo con la clasificación de la OMS, el valor de la media se encuentra dentro de la categoría de sobrepeso $\left(25.5-29.9 \mathrm{~kg} / \mathrm{m}^{2}\right)$.
Asimismo, el $47.1 \%$ de la población presentó obesidad. Respecto a la escolaridad de los participantes, el grupo de referencia (no expuesto a plaguicidas de manera ocupacional) tuvo un mayor número de años de estudio (14.7) vs. los grupos expuestos a plaguicidas (11.5). En cuanto a los hábitos nocivos, el $21.6 \%$ de los participantes eran fumadores activos y $77.4 \%$ fumadores pasivos, $86.3 \%$ consumían alcohol y $5.8 \%$ refirieron haber consumido algún tipo de droga (Cuadro I).

En el Cuadro II se presenta la información referente al uso de equipo de protección personal de los aplicadores de plaguicidas. Tanto el grupo de exposición moderada como el de exposición alta refirieron no utilizar de manera rutinaria equipo de protección personal como overol, cubre bocas, guantes, botas, lentes de seguridad u otros. En el caso de sombrero o gorra, ambos grupos refirieron usarlos siempre.

Las actividades realizadas por los 163 participantes que contestaron esta sección indican que el 31.3 $\%$ llevan a cabo actividades de abatización, mientras que el $63.2 \%$ realizaba tanto la mezcla, como la aplicación y entrega de plaguicidas. Por otra parte, dentro de los métodos que utilizaban para la aplicación de plaguicidas se encuentra el uso de vehículo con bomba, mochila con bomba, motobomba, así como aplicación con la mano (Cuadro III).

De acuerdo con la acción específica, los larvicidas e insecticidas son los plaguicidas más utilizados por los trabajadores, y en menor proporción se utilizan los herbicidas (Cuadro IV).

Por otra parte, respecto al patrón de uso de plaguicidas utilizados por la población de estudio (Figs. 1 y 2), los organofosforados (OF) son los más utilizados $(63.3 \%)$, seguidos de los piretroides $(29.8 \%)$ y carbamatos $(3.8 \%)$. Dentro de los OF, un alto porcentaje de participantes refirió utilizar temefos y clorpirifos. En cuanto a los piretroides, los más utilizados fueron deltametrina y permetrina-esbioaletrina, mientras que de los carbamatos el más utilizado fue bendiocarb. Otros plaguicidas utilizados por la población de estudio fueron glifosato y 2,4-D. Un aspecto importante es que de acuerdo con la clasificación de la OMS (basada en su nivel de toxicidad aguda), el 6.7 y el $13.3 \%$ del total de los plaguicidas utilizados se clasifican como extremadamente (Ia) y altamente peligrosos (Ib), respectivamente. Asimismo, es importante destacar que algunos fumigadores urbanos también realizaban actividades de fumigación en campos agrícolas, lo que puede explicar el uso de compuestos como glifosato y 2,4-D.

En el Cuadro V se muestra la información relacionada con la presentación en la que se utilizan los 
CUADRO I. CARACTERÍSTICAS ANTROPOMÉTRICAS Y DE HÁBITOS NOCIVOS EN LA POBLACIÓN DE ESTUDIO

\begin{tabular}{|c|c|c|c|c|}
\hline Características & $\begin{array}{l}\text { Grupo de referencia } \\
\qquad n=22\end{array}$ & $\begin{array}{l}\text { Grupo de exposición } \\
\text { moderada } n=126\end{array}$ & $\begin{array}{c}\text { Grupo de exposición } \\
\text { alta } n=60\end{array}$ & Valor de $p$ \\
\hline Género & & & & 0.09 \\
\hline Mujeres [n (\%)] & $5(32)$ & $49(39.5)$ & $15(25)$ & \\
\hline Hombres $[\mathrm{n}(\%)]$ & $17(68)$ & $77(60.5)$ & $45(75)$ & \\
\hline Edad $*$ (rango) & $34.9(20-68)$ & $37.3(20-67)$ & $34.7(20-57)$ & 0.16 \\
\hline $\mathrm{IMC}^{* \pm}$ (rango) & $28.6(20.1-39.9)$ & $29.5(19.0-45.5)$ & $28.8(20.4-54.4)$ & 0.25 \\
\hline Bajo peso & - & - & - & - \\
\hline Peso normal (\%) & $5(22.7)$ & $18(14.3)$ & $15(25.0)$ & $<0.001$ \\
\hline Sobrepeso $(\%)$ & $8(36.4)$ & $43(34.1)$ & $21(35.0)$ & $<0.001$ \\
\hline Obesidad $(\%)$ & $9(40.9)$ & $65(51.6)$ & $24(40.0)$ & $<0.001$ \\
\hline Escolaridad (años de estudio)* & $14.7(9-19)$ & $11.48(5-19)$ & $11.50(8-19)$ & $<0.001$ \\
\hline Fumadores activos [n (\%)] & $8(36.4)$ & $18(15.08)$ & $19(31.67)$ & 0.009 \\
\hline Fumadores pasivos $[\mathrm{n}(\%)]$ & $21(95.4)$ & $90(73.77)$ & $50(84.75)$ & 0.03 \\
\hline Consumo de alcohol [n (\%)] & $20(90.9)$ & $105(83.33)$ & $55(91.66)$ & 0.22 \\
\hline Consumo de drogas $[\mathrm{n}(\%)]$ & - & $7(5.56)$ & $5(8.33)$ & 0.35 \\
\hline Actividad BuChE (IC $95 \%$ ) & $\begin{array}{c}6605.1 \\
(6194.5-7042.1)\end{array}$ & $\begin{array}{c}5691.8 \\
(5372.8-6029.8)\end{array}$ & $\begin{array}{c}4178.2 \\
(3863.8-4518.1)\end{array}$ & $<0.001$ \\
\hline
\end{tabular}

* Los valores se presentan como medias geométricas. IC $95 \%$, intervalo de confianza al $95 \%$. ${ }^{ \pm}$Clasificación según la OMS: bajo peso $\leq 18.5$; rango normal $=18,5-24,99$; sobrepeso $=25-29,99$; obesidad $\geq 30$

plaguicidas por la población de estudio. De acuerdo con estos datos, el $44.4 \%$ utiliza presentaciones en polvo y líquidos.

\section{Conocimientos, percepción y prácticas sobre pla- guicidas de la población de estudio}

De los 208 individuos, el $11.1 \%$ se ha intoxicado en alguna ocasión y el $69.1 \%$ manifestó saber lo que significa una intoxicación por plaguicidas.

Con la finalidad de conocer el tipo de capacitación que reciben estos trabajadores, se les preguntó sobre los temas principales de las pláticas que reciben en sus programas de instrucción inherentes a la temática de plaguicidas. El $4.2 \%$ refirió haber recibido pláticas correspondientes a los tipos de plaguicidas, $9.8 \%$ sobre el buen uso de estos compuestos, $2.8 \%$ sobre cómo protegerse adecuadamente, $2.8 \%$ sobre mantenimiento y uso de equipo, $2.1 \%$ sobre daños a la salud por el uso de plaguicidas y $75.5 \%$ sobre todos los temas antes descritos. Menos del $3.0 \%$ mencionó no haber recibido información sobre la protección adecuada en el uso y manejo de plaguicidas o temas referentes a daños a la salud ocasionados por el contacto con los mismos. Si bien el $60.8 \%$ mencionó conocer el significado de las etiquetas en los envases, el $36.7 \%$ no las lee y el $10 \%$ de la población no sabe la dosis que debe usar de acuerdo con el plaguicida.

En la Figura 3 se presentan los resultados referentes al manejo y uso de estos compuestos. Los datos sugieren que el $20.9 \%$ de los fumigadores se cambian de ropa hasta que llega la noche (después de terminar su jornada laboral), el $20.0 \%$ manifestó salpicarse siempre al manejar o hacer uso de plaguicidas y el 8.6 $\%$ ha almacenado equipo de protección o plaguicidas en su casa. El $47.8 \%$ de los fumigadores ingiere alimentos durante la aplicación de plaguicidas y el 6.7 $\%$ de los participantes fuma durante la aplicación de los mismos. Más del $60 \%$ de esta población manifestó lavar los equipos de aplicación de plaguicidas y estar o haber estado en contacto anteriormente con estos compuestos. Finalmente, es de destacarse que el $33.3 \%$ de los encuestados no percibe a su ocupación como peligrosa para su salud.

\section{DISCUSIÓN}

\section{Tipo de plaguicidas utilizados en la zona de es- tudio}

Con base en el patrón de uso de plaguicidas registrado en este estudio, se encontró que la familia de OF sigue siendo la más utilizada para estas actividades, en presentaciones de polvos y líquidos. Los resultados de esta investigación son consistentes con otros reportados en la literatura (Durán-Nah y CollíQuintal 2000, Pose et al. 2000, García et al. 2002, Altamirano et al. 2004, Hernández et al. 2007, RojasGarcía et al. 2011). En un estudio realizado en Nayarit, González-Arias et al. (2010) encontraron que los 

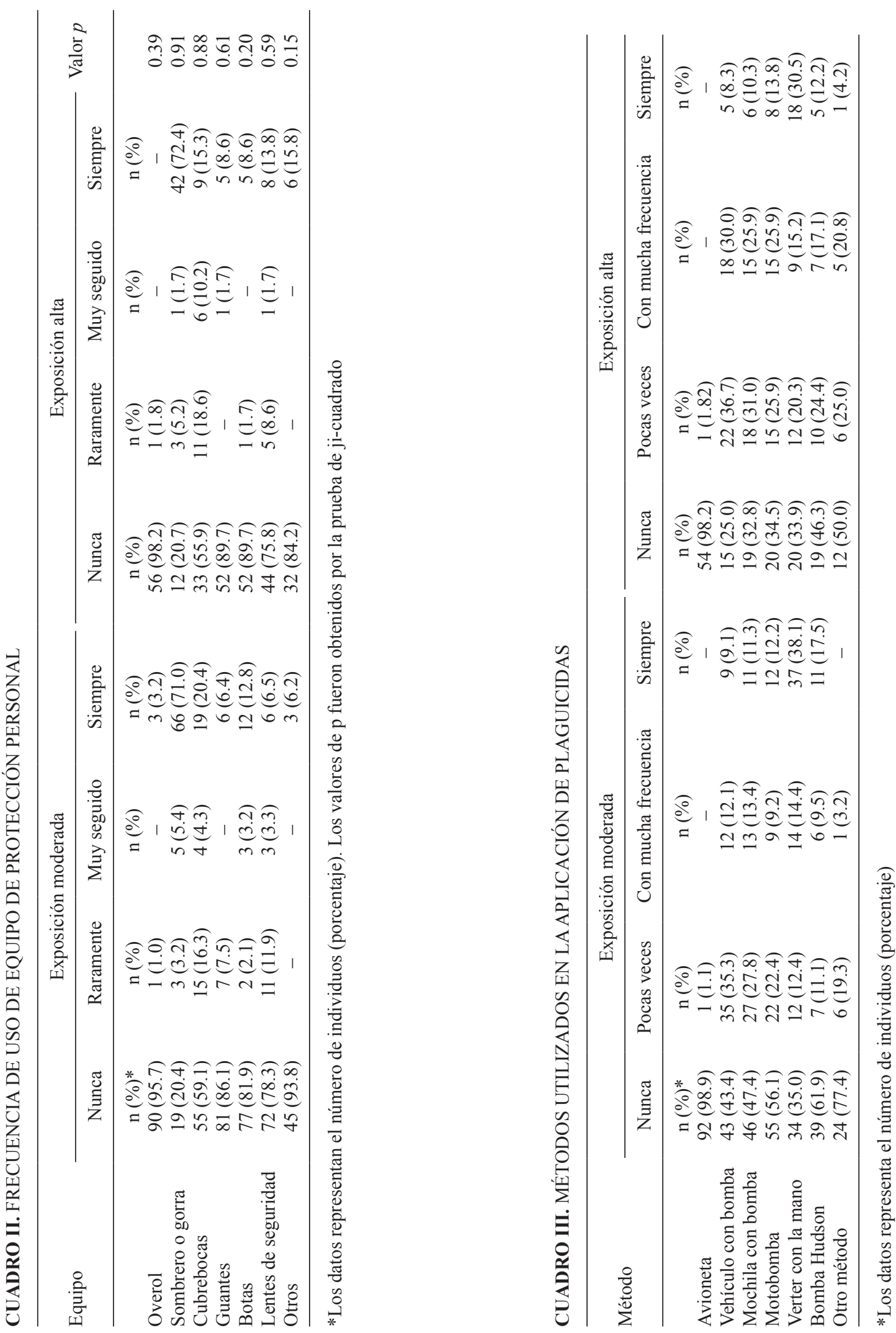
CUADRO IV. TIPOS DE PLAGUICIDA UTILIZADOS POR ACCIÓN ESPECÍFICA

\begin{tabular}{lrc}
\hline & $\mathrm{n}$ & Porcentaje \\
\hline Herbicidas & 1 & 0.6 \\
Insecticidas & 4 & 2.5 \\
Larvicidas & 36 & 22.5 \\
Insecticidas y larvicidas & 89 & 55.6 \\
Otro tipo de plaguicidas & 2 & 1.2 \\
Desconoce el tipo de plaguicida & 1 & 0.6 \\
\hline
\end{tabular}

$\mathrm{n}=$ participantes que respondieron esta sección de la encuesta. $\mathrm{n}$ total $=160$

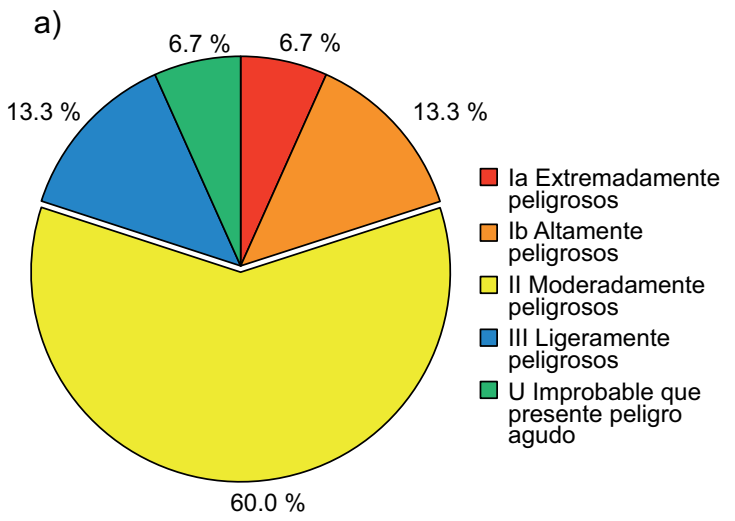

CUADRO V. PRESENTACIÓN EN LA QUE SE UTILIZAN LOS PLAGUICIDAS

\begin{tabular}{lcc}
\hline Presentación & $\mathrm{n}$ & Porcentaje \\
\hline Sólo polvos & 49 & 30.6 \\
Sólo líquidos & 13 & 8.1 \\
Polvos y líquidos & 71 & 44.4 \\
Polvos y líquidos mezclados & 25 & 15.6 \\
Polvos y líquidos/otros & 2 & 1.2 \\
\hline
\end{tabular}

$\mathrm{n}=$ participantes que respondieron esta sección de la encuesta. $\mathrm{n}$ total $=160$

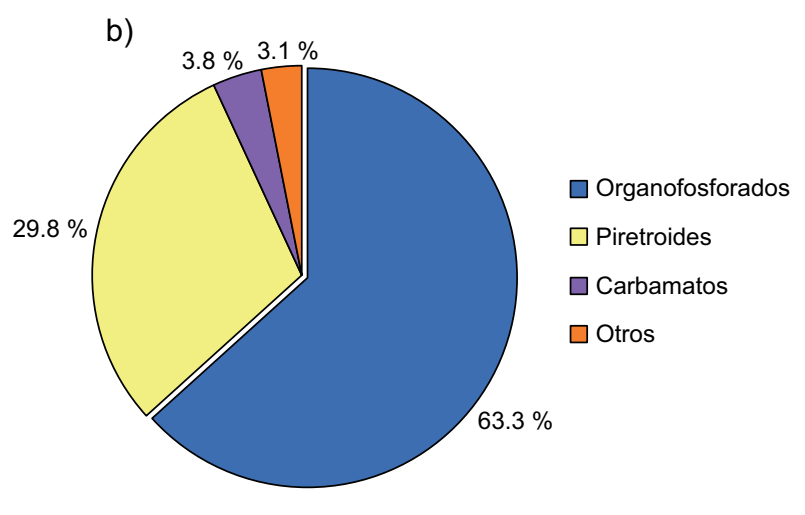

Fig. 1. Frecuencia de uso de plaguicidas de acuerdo con (a) la clasificación de la OMS y (b) el grupo químico

plaguicidas más utilizados y comercializados son los OF $(52.0 \%)$, seguidos de los piretroides $(26.4 \%)$, carbamatos $(13.2 \%)$ y organoclorados $(8.4 \%)$. Nuestros resultados muestran que el plaguicida OF más utilizado por la población fue temefos. Aunque este plaguicida es clasificado por la OMS (2009) dentro de la categoría III, es decir, improbable que presente toxicidad aguda, es importante considerar que en un estudio realizado por Benítez-Trinidad et al. (2015) se reportó que este compuesto produce daño genotóxico permanente en la línea celular de hepatocarcinoma humano (HepG2), por lo que es necesario propiciar buenas prácticas de manejo y uso de este tipo de plaguicidas que son aplicados de manera manual. Otro aspecto importante a destacar es que, de acuerdo con la clasificación de la OMS, el $20 \%$ de los plaguicidas usados por esta población son OF clasificados como extremadamente y altamente peligrosos (Ia y $\mathrm{Ib}$, respectivamente).

En este estudio el $11.1 \%$ de los participantes se ha intoxicado por lo menos en una ocasión y el 30.9 \% refirió no conocer qué es una intoxicación. Estas variables son similares a las observadas en un estudio realizado por López et al. (2008) en el que se encontró que el $29.5 \%$ de la población de estudio se ha intoxicado en alguna ocasión y el $22.0 \%$ de los individuos ocupacionalmente expuestos expresaron no conocer qué es una intoxicación. Las variables anteriores, junto con otras como el conocimiento del significado de las etiquetas de los envases, la dosis a usar de acuerdo al área y haber recibido capacitaciones para el uso de plaguicidas, son fundamentales para conocer la percepción del riesgo de estas poblaciones (Pernalete y Hernández 2016). Es importante considerar que estas variables aportan información del conocimiento que tienen los participantes $\mathrm{y}$, sin duda, impactan en su percepción del riesgo. Sin embargo, hay otros factores que determinarán esta percepción, como se ha mencionado anteriormente.

\section{Prácticas en el manejo y uso de plaguicidas}

Se ha señalado que la brecha entre el conocimiento y la práctica del personal ocupacionalmente expuesto de forma directa o indirecta, necesita superarse mediante modelos de capacitación más interactivos y participativos que ayuden a reducir los impactos sobre la salud de los trabajadores y el ambiente, y originen un cambio en el comportamiento de los tra- 

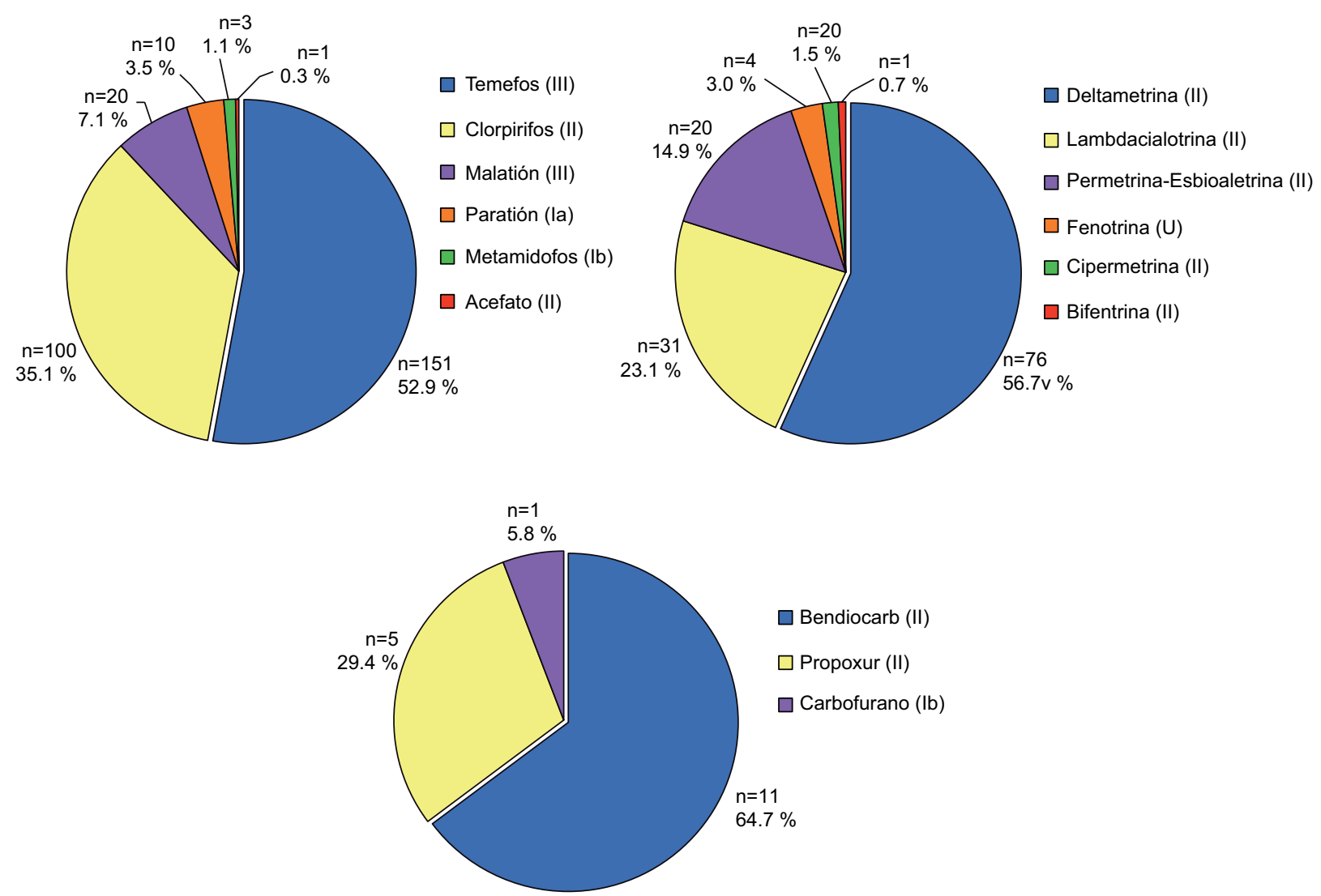

Fig. 2. Patrón de uso de plaguicidas (a) organofosforados, (b) piretroides y (c) carbamatos. La peligrosidad de las clases, de acuerdo con la clasificación de la OMS, es la siguientes: Ia, extremadamente peligrosos; Ib, altamente peligrosos; II, moderadamente peligrosos; III, ligeramente peligrosos; $\mathrm{U}$, improbable que presente peligro agudo

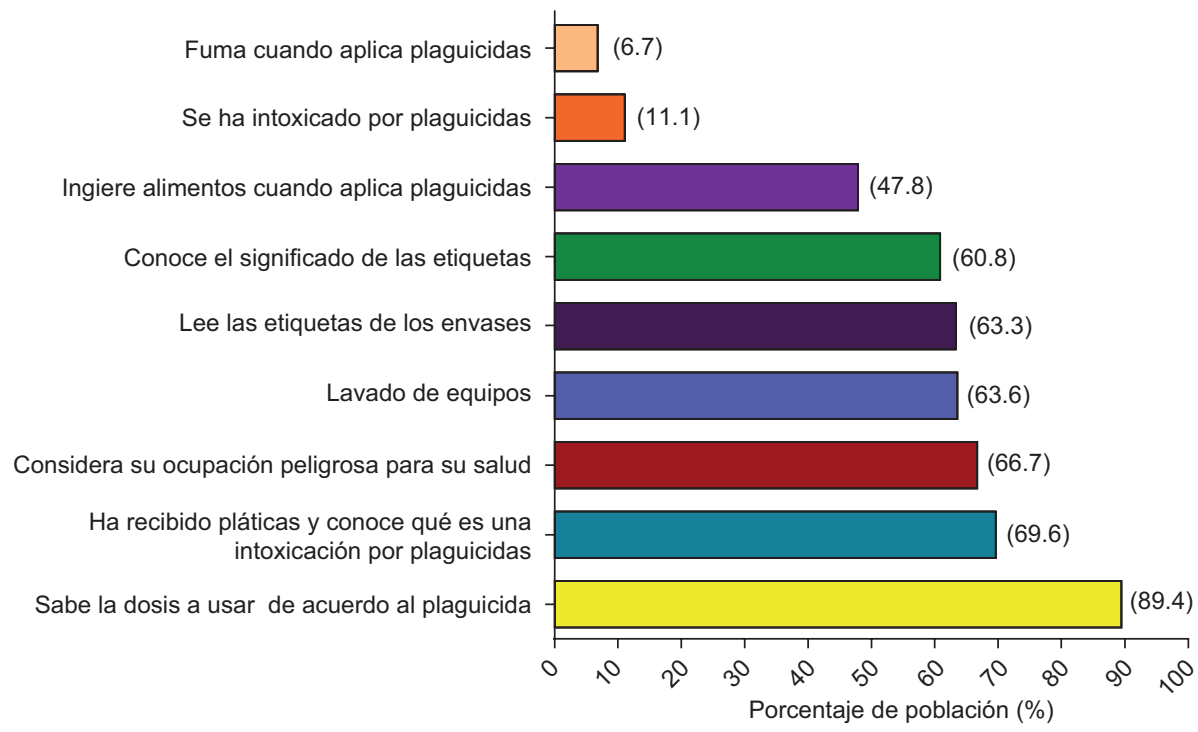

Fig. 3. Prácticas de manejo y uso de plaguicidas por la población de estudio 
bajadores para realizar sus actividades de una manera más sana y segura (López et al. 2008, Calliera et al. 2013, Yuantari et al. 2015, Houbraken et al. 2016). Por otro lado, algunos estudios mencionan el escaso conocimiento del riesgo por exposición a este tipo de compuestos en poblaciones dedicadas a las actividades de fumigación (Karunamoorthi et al. 2011, Hlongwana et al. 2013, Lekei et al. 2014, Gesesew et al. 2016, Pernalete y Hernández 2016).

Con relación a lo anterior, en el presente trabajo se destaca que a pesar de que la mayoría de los encuestados refirieron haber recibido pláticas sobre plaguicidas a través de programas de capacitación, se siguen realizando prácticas consideradas como factores de riesgo de exposición. Los resultados señalan que el baño y cambio de ropa se realiza hasta llegar a su casa y no inmediatamente después de las actividades de fumigación. En otros estudios se ha reportado que dicha práctica es un factor de riesgo de exposición (García et al. 2002, Hernández et al. 2007, Al-zain y Mosalami 2014).

En este contexto, otra conducta observada en la población de estudio fue el almacenamiento de plaguicidas o equipos de protección en su casa. Esta práctica igualmente se ha señalado en otros estudios que han evaluado conductas de riesgo de exposición a plaguicidas (Hernández et al. 2007, Lekei et al. 2014, Houbraken et al. 2016). Ingerir alimentos y fumar durante la aplicación de plaguicidas son conductas que también pueden aumentar la exposición a estos xenobióticos (López et al. 2008, Hlongwana et al. 2013, Al-zain y Mosalami 2014, Gesesew et al. 2016) y que fueron referidas por los encuestados.

La exposición de los trabajadores dedicados a las actividades de fumigación puede incrementarse cuando no cuentan con conocimientos sobre medidas de seguridad adecuadas, o bien tienen el conocimiento pero no cuentan con el equipo de protección o la voluntad de usarlo. En el caso concreto del uso de equipo de protección personal, diversos estudios han evidenciado la falta de su adecuada utilización o poco uso; en este sentido, los participantes de este trabajo (grupos de moderada y alta exposición), refieren no utilizarlos de manera rutinaria. Lo que sí utilizan es un sombrero o gorra. Estos resultados son consistentes con el estudio realizado por Hashemi et al. (2012) en el que $60 \%$ de una población de individuos expuestos ocupacionalmente a plaguicidas refirió no usar equipo de protección al realizar actividades de fumigación. En este estudio, el $73.6 \%$ de la población manifestó no utilizar equipo de protección personal. De la misma manera, son diversas las investigaciones que refieren a poblaciones que no utilizan el equipo de protección por cuestiones de idiosincrasia, o bien porque las condiciones climatológicas no lo favorecen (Damalas et al. 2006, Recena et al. 2006, MacFarlane et al. 2008, Rojas-García et al. 2011). Es importante conducir más estudios encaminados a evaluar de manera extensa, los motivos por los que poblaciones mexicanas ocupacionalmente expuestas a plaguicidas no utilizan el equipo de protección personal.

Todas estas prácticas de riesgo sugieren - como han señalado otros autores - que la prevención del riesgo por exposición a plaguicidas requiere un análisis sistemático que involucre la evaluación, percepción, comunicación y manejo del riesgo que producen estos xenobióticos (Maroni et al. 1999), puesto que una baja percepción del riesgo en poblaciones laboralmente expuestas a plaguicidas puede considerarse un factor importante en las tasas de intoxicación (Gómez y Cáceres 2010).

Es de suma importancia que los trabajadores dedicados a las actividades de fumigación identifiquen el peligro, conozcan las prácticas que pueden aumentar su exposición y los efectos deletéreos a la salud ocasionados por su ocupación. Si bien hay estudios en la literatura que reportan el escaso conocimiento del riesgo (López et al. 2008, Pernalete y Hernández 2016), es importante considerar dentro de los programas de prevención variables relacionadas con la percepción del riesgo que vayan más allá de la evaluación de reactivos inherentes al conocimiento, es decir, que no sólo evalúen las prácticas sino también las conductas en el manejo y uso de plaguicidas. En este contexto, Slovic y Weber (2002) sugieren el papel complejo que pueden jugar las emociones y razones dentro de la percepción del riesgo (enfoque psicométrico); sin embargo, también es relevante considerar metodologías cualitativas dentro de las cuales se contemplen las perspectivas socioculturales.

Debido a que la población de estudio no realiza prácticas adecuadas durante su jornada laboral, el riesgo de que se presenten efectos adversos en la salud aumenta. Un estudio realizado por Monier et al. (2010) en individuos que utilizan plaguicidas para el control del mosquito Aedes aegypti mostró una falta de satisfacción en los trabajadores respecto a la ausencia de capacitación y mala organización del trabajo. En este estudio no se evaluó la satisfacción de los trabajadores respecto de los programas de capacitación relacionados con el uso y manejo de plaguicidas, lo que consideramos fue una de sus limitaciones.

Finalmente, aunque no directamente relacionado con la exposición a este tipo de agentes, pero si como un problema de salud pública que debe atenderse 
en la zona de estudio, se destaca que más del $75 \%$ de la población encuestada presentó sobrepeso u obesidad. Estos datos son importantes porque un aumento en el IMC se asocia con alteraciones cardiovasculares (principalmente cardiopatías y accidentes cerebrovasculares), diabetes, trastornos del aparato locomotor (osteoartritis, enfermedad degenerativa de las articulaciones) y algunos tipos de cáncer (endometrio, mama, ovarios, próstata, hígado, vesícula biliar, riñones y colon) (OMS 2016). Los datos obtenidos son similares a los reportados en la encuesta nacional de salud y nutrición en México de 2012, en la que adultos de 20 años o más tuvieron una prevalencia combinada de sobrepeso y obesidad de 71.2 $\%$ (IC95\% 70.5, 72.1) (ENSANUT 2012), mientras que en 2016 esta prevalencia fue de $72.5 \%$ (IC95\% 70.8, 74.3) (ENSANUT 2016).

\section{CONCLUSIONES}

Los resultados de este estudio muestran que los principales plaguicidas utilizados en actividades de fumigación pertenecen a los OF y piretroides, que el $11.1 \%$ de los trabajadores que los emplean han sufrido al menos una intoxicación aguda por plaguicidas y el $33.3 \%$ no considera su ocupación peligrosa para su salud. Asimismo, estos datos sugieren la necesidad de una capacitación técnica adecuada para el personal encargado de la manipulación de plaguicidas, la implementación de protocolos de vigilancia, exámenes médicos y de laboratorio, así como estudios encaminados no sólo a evaluar las prácticas del uso y manejo de estos compuestos, sino además la percepción del riesgo en poblaciones ocupacionalmente expuestas a estos xenobióticos.

\section{AGRADECIMIENTOS}

Los autores agradecen a la Red Temática de Toxicología de Plaguicidas (CONACyT$262284 / 280045 / 294303$ ) y a los participantes de este estudio. Este trabajo fue financiado por el Fondo SS/ IMSS/ISSSTE-CONACyT-233803.

\section{REFERENCIAS}

Altamirano J.E., Franco R. y Bovi Miltre M.G. (2004). Modelo epidemiológico para el diagnóstico de intoxicación aguda por plaguicidas. Revista de Toxicología 21(2-3), 98-102.
Al-zain B.F. y Mosalami J. (2014). Pesticides usage, perceptions, practices and health effects among farmers in North Gaza, Palestine. Indian J. Appl. Res. 4, 17-22.

Arellano-Aguilar O. y Rendón V.O.J. (2016). La huella de los plaguicidas en México. Greenpeace [en línea]. http://m.greenpeace.org/mexico/Global/mexico/ Graficos/2016/comida-sana/Plaguicidas_en_agua_ ok_EM.pdf

Benítez-Trinidad A.B., Herrera-Moreno J.F., VázquezEstrada G., Verdín-Betancourt F.A., Sordo M., Ostrosky-Wegman P., Bernal-Hernández Y.Y., MedinaDíaz I.M., Barrón-Vivanco B.S., Robledo-Marenco M.L., Salazar A.M. y Rojas-García A.E. (2015). Cytostatic and genotoxic effect of temephos in human lymphocytes and HepG2 cells. Toxicol. In Vitro 29(4), 779-86.

DOI: 10.1016/j.tiv.2015.02.008

Benítez-Trinidad A.B., Herrera-Moreno J.F., XotlanihuaGervacio M.C., Bernal-Hernández Y.Y., Medina-Díaz I.M., Barrón-Vivanco B.S., González Arias C.A. y Rojas-García A.E. (2018). Patrón de uso de plaguicidas y biomarcadores bioquímicos en una población de fumigadores urbanos. Rev. Int. Contam. Ambie. 34 (CTP) 61-71.

DOI: 10.20937/RICA.2018.34.esp01.04

Berube D.M. (2009). Intuitive toxicology: The public perception of nanoscience. En: Nanotechnology and society (F. Allhoff y P. Lin Eds.). Springer Netherlands, pp. 91-108. DOI: 10.1007/978-1-4020-6209-4

Calliera M., Berta F., Galassi T., Mazzini F., Rossi R., Bassi R., Meriggi P., Bernard A., Marchis A., Di Guardo A. y Capri E. (2013). Enhance knowledge on sustainable use of plant protection products within the framework of the sustainable use directive. Pest. Manag. Sci. 69, 883-888. DOI: $10.1002 / p s .3579$

COFEPRIS (2015). Plaguicidas y fertilizantes. Catálogo de plaguicidas. Comisión Federal para la Protección contra Riesgos Sanitarios. [en línea]. http://www. cofepris.gob.mx/AZ/Paginas/Plaguicidas $\% 20 y \% 20$ Fertilizantes/CatalogoPlaguicidas.aspx 05/01/2017

Damalas C.A., Georgiou E.B. y Theodorou M.G. (2006). Pesticide use and safety practices among Greek tobacco farmers: A survey. Int. J. Environ. Health Res. 16, 339-348. DOI: 10.1080/09603120600869190

Durán-Nah J.J. y Collí-Quintal J. (2000). Intoxicación aguda por plaguicidas. Salud Pública Mex. 42, 53-55.

ENSANUT (2012). Encuesta Nacional de Salud y Nutrición. Instituto Nacional de Salud Pública, México [en línea]. http://www.ensanut.insp.mx/

ENSANUT (2016). Encuesta Nacional de Salud y Nutrición. Instituto Nacional de Salud Pública, México [en línea]. http://www.ensanut.insp.mx/ensanut2016/ index.php 
FAO (2014). Código internacional de conducta para la gestión de plaguicidas. Actualización. Food and Agriculture Organization of the United Nations. Código, Roma, Italia, 6 pp.

FAO (2016). Pesticides use. FAOSTAT. Food and Agriculture Organization of the United Nations. [en línea]. http://www.fao.org/faostat/en/\#data/RP 14/01/2017

García A.M., Ramírez A. y Lacasaña M. (2002). Prácticas de utilización de plaguicidas en agricultores. Gac. Sanit. 16, 236-240. DOI: 10.1016/S0213-9111(02)71667-1

Gesesew H.A., Woldemichael K., Massa D. y Mwanri L. (2016). Farmers knowledge, attitudes, practices and health problems associated with pesticide use in rural irrigation villages, Southwest Ethiopia. PLoS One 13, 9: e0162527. DOI: 10.1371/journal.pone.0162527.

Gómez M.J. y Cáceres J.L. (2010). Toxicidad por insecticidas organofosforados en fumigadores de campaña contra el dengue, estado Aragua, Venezuela. Bol. Mal. Salud Amb. 50, 119-125.

González-Arias C.A., Robledo-Marenco M.L., MedinaDíaz I.M., Velázquez-Fernández J.B., Girón-Pérez M.I., Quintanilla-Vega B., Ostrosky-Wegman P., PérezHerrera N.E. y Rojas-García A.E. (2010). Patrón de uso y venta de plaguicidas en Nayarit, México. Rev. Int. Contam. Ambie. 26, 221-228.

Hashemi S.M., Rostami R., Hashemi M.K. y Damalas C.A. (2012). Pesticide use and risk perceptions among farmers in Southwest Iran. Hum. Ecol. Risk Assess. 18, 456-470. DOI: 10.1080/10807039.2012.652472

Hernández G.M.M., Jiménez G.C., Jiménez A.J. y Arceo G.M.E. (2007). Caracterización de las intoxicaciones agudas por plaguicidas: perfil ocupacional y conductas de uso de agroquímicos en una zona agrícola del Estado de México, México. Rev. Int. Contam. Ambie. 23, 159-167.

Hlongwana K.W., Mavundza E.J., Mohapi E.P., Kruger P., Urbach J., Mukaratirwa S. y Maharaj R. (2013). Vector-control personnel's knowledge, perceptions and practices towards insecticides used for indoor residual spraying in Limpopo Province, South Africa. Parasite. Vector. 23, 118. DOI: 10.1186/1756-3305-6-118.

Houbraken M., Bauweraerts I., Fevery D., Van Labeke M.C. y Spanoghe P. (2016). Pesticide knowledge and practice among horticultural workers in the Lâm Đồng region, Vietnam: A case study of chrysanthemum and strawberries. Sci. Total Environ. 15, 1001-1009.

DOI: 10.1016/j.scitotenv.2016.01.183

Karunamoorthi K., Mohammed A. y Jemal Z. (2011). Peasant Association Member's knowledge, attitudes, and practices towards safe use of pesticide management. Am. J. Ind. Med. 54, 965-970. DOI: 10.1002/ ajim.21008.
Lekei E.E., Ngowi A.V. y London L. (2014). Farmers' knowledge, practices and injuries associated with pesticide exposure in rural farming villages in Tanzania. BMC Public Health 23, 389.

DOI: 10.1186/1471-2458-14-389.

López N.L., López B.I., Carrero P.R.A., Cerda R.A.M. y Cruz B.C.M. (2008). Conocimientos, actitudes y práctica del uso y manejo de plaguicidas por pequeños productores de los municipios de Chinandega, en el período Septiembre-Octubre del 2006. Universitas 1, 51-57.

Macfarlane E., Chapman A. Benke G., Meaklim J, Sim M. y McNeil J. (2008). Training and other predictors of personal protective equipment use in Australian grain farmers using pesticides. Occup. Environ. Med. 65, 141-146. DOI: 10.1136/oem.2007.034843

March G.J. (2014). Producción agrícola y uso de plaguicidas. En: Agricultura y plaguicidas, un análisis global. (Efe y Efe, Eds.). FADA-Fundación Agropecuaria para el Desarrollo de Argentina. Río Cuarto, Córdoba Argentina, pp. 23-45.

Maroni M., Fait A. y Colosio C. (1999). Risk assessment and management of occupational exposure to pesticides. Toxicol. Lett. 107, 145-53.

DOI: $10.1016 / \mathrm{S} 0378-4274(99) 00041-7$

Martínez-Valenzuela C. y Gómez-Arroyo S. (2007). Riesgo genotóxico por exposición a plaguicidas en trabajadores agrícolas. Rev. Int. Contam. Ambient. 23, $185-200$.

Monier A., Gilart N. y Sanchez I. (2010). Evaluación de la satisfacción de los operarios de la campaña antivectorial contra el Aedes aegypti. MEDISAN. 14, 761-766.

OMS (2016). BMI classification. Global database on body mass index. Organización Mundial de la Salud [en línea]. http://apps.who.int/bmi/index. jsp?introPage $=$ intro_3.html 15.12.2016

OMS (2009). The WHO recommended classification of pesticides by hazard and guidelines to classification 2009. Organización Mundial de la Salud [en línea] http://apps.who.int/iris/bitst ream/10665/44271/1/9789241547963_eng.pdf $27 / 12 / 2016$

OPS/OMS (2002). Situación epidemiológica de las intoxicaciones agudas por plaguicidas en el Istmo centroamericano. Organización Panamericana de la Salud/Organización Mundial de la Salud. Boletín Epidemiológico 23(3) [en línea]. http://cidbimena.desastres.hn/docum/ops/publicaciones/EpidemiologicO/ EB_v23n3.pdf

Pernalete R.M.E. y Hernández P.A.J. (2016). Riesgos laborales por exposición a plaguicidas contra el mosquito Aedes aegypti. SABER, Universidad de Oriente, Venezuela 28, 5-17. 
Pose D., De Benz S., Delfîno N., Burger M. (2000). Intoxicación aguda por organofosforados. Factores de riesgo. Rev. Med. Uruguay 16, 5-13.

Recena M.C., Caldas E.D., Pires D.X. y Pontes E.R. (2006). Pesticides exposure in Culturama, Brazilknowledge, attitudes, and practices. Environ. Res. 102, 230-236.

DOI: 10.1016/j.envres.2006.01.007

Rojas-García A.E., Medina-Díaz I.M., Robledo-Marenco M. L., Barrón-Vivanco B.S., Girón-Pérez M.I., Velázquez-Fernández J.B., González-Arias C.A., Albores-Medina A., Quintanilla-Vega B., OstroskyWegman P., Rojas García M.C., Pérez-Herrera N.E. y López-Flores J.F. (2011). Hematological, biochemical effects, and self-reported symptoms in pesticide retailers. J. Occup. Environ. Med. 53, 517-21. DOI: 10.1097/JOM.0b013e318215fbf2.

Sánchez-González J.M., Ramos-Remus C., Jácome-Sánchez B., García-Ortíz R., Flores-Ramos J. y Santoscoy H. F. (2016). Virus Zika en México. Rev. Latinoam. Patol. Clin. Med. Lab. 63 (1), 4-12.
Sánchez-Guerra M., Pérez-Herrera N. y Quintanilla-Vega B. (2011). Organophosphorous pesticides research in Mexico: epidemiological and experimental approaches. Toxicol. Mech. Methods. 21, 681-691. DOI: $10.3109 / 15376516.2011 .602130$.

Slovic P. y Weber E.U. (2002). Perception of risk posed by extreme events. En: Regulation of Toxic Substances and Hazardous Waste (2a Ed.) (Applegate J., Laitos J., Gaba J., y Sachs N., Eds.). Foundation Press (forthcoming). Disponible en: SSRN: https://ssrn.com/ abstract $=2293086$

Worek F., Mast U., Kiderlen D., Diepold C., Eyer P. (1999). Improved determination of acetylcholinesterase activity in human whole blood. Clinica Chimica Acta 288, 73-90.

Yuantari M.G.C., Van Gestel C.A.M., Van Straalen N.M., Widianarko B., Sunoko H.R. y Shobib M.N. (2015). Knowledge, attitude, and practice of Indonesian farmers regarding the use of personal protective equipment against pesticide exposure. Environ. Monit. Assess. 187 (3), 142. DOI: 10.1007/s10661-015-4371-3. 